https://doi.org/10.15407/ujpe63.10.924

A.D. SUPRUN, L.V. SHMELEVA

Taras Shevchenko National University of Kyiv

(64/13, Volodymyrs'ka Str., Kyiv 01601, Ukraine; e-mail: lshmel@univ.kiev.ua)

\title{
CENTROSYMMETRIC SOLITONS WITH POWER ASYMPTOTICS FOR MEDIA OF DIFFERENT DIMENSIONS
}

\begin{abstract}
Analytic solutions of radially symmetric nonlinear Schrödinger equations with two nonlinear terms with different powers are analyzed for 1D, 2D, and 3D spaces. They are typical of the equations, where there are two nonlinear terms instead of one cubic term, as a rule. An important feature of the solutions obtained is that they are expressed not in terms of hyperbolic functions, but in terms of rational functions finite in the entire space with a power asymptotics at infinity. The solutions obtained significantly expand the range of applications of the nonlinear Schrödinger equations. Separate relevant cases of the general solution are considered in the applications.
\end{abstract}

Ke ywords: centrosymmetric solitons, nonlinear Schrödinger equation, graphene.

\section{Introduction}

Nonlinear Schrödinger equations have a wide range of applications from polymers [1] and polymer-like systems $[2,3]$ to solids [4-9] and a plasma $[10,11]$. Despite this, there are still a number of problems that require to be solved. Till the recent time, one of such problems was the lack of analytical solutions to the radially symmetric nonlinear Schrödinger equation. Except the numerical and asymptotic solutions $[12,13]$, it is worth to mention the studies carried out to find analytical solutions [4-7]. In [4-6], a radially symmetric modified nonlinear Schrödinger equation with a nonlinearity of differential-degree nature in a $2 \mathrm{D}$ space is considered $[4,5]$, and the analysis of localized states [6] in the $2 \mathrm{D}$ space and in the quasimomentum representation is carried out. There was found the case [7], which admits an analytic solution for the radially symmetric nonlinear Schrödinger equation. Radially symmetric soliton solutions in two-dimensional systems are now especially relevant due to the wide application of hyperfine films (sev-

(C) A.D. SUPRUN, L.V. SHMELEVA, 2018

924 eral atomic layers) [14] and graphenes [8]. On the other hand, the one-dimensional systems also do not lose relevance. They have well-known soliton solutions [15], which are expressed in terms of hyperbolic functions.

We will analyze a radially symmetric modified nonlinear Schrödinger equation with complex power nonlinearity and zero boundary conditions at infinity. In contradistinction to the modified Schrödinger equation considered in [4-6], we consider a nonlinearity consisting of two terms, each of which contains the unknown function in various powers. Such nonlinearity is usually a result of the expansion, for example, of some potential in a series with the preservation of two terms (usually only the first non-vanishing term is conserved in such expansions). In addition, we will introduce a parameter into the equation that allows us to simultaneously analyze this radially symmetric problem for all three spatial dimensions: 1D (polymers), 2D (graphene, etc.) and 3D (objects of the crystal type).

The problem under consideration is relevant due to the search for radially symmetric soliton-type solu-

ISSN 2071-0194. Ukr. J. Phys. 2018. Vol. 63, No. 10 
tions in nonlinear optics, nonlinear quantum mechanics, and nonlinear solid-state theory (or condensed matter). It can also be relevant in studies of the interaction of a plasma with electromagnetic radiation. It should be noted that the solutions found, strictly speaking, are not solitons, although they are localized solutions of stationary nonlinear equations. They do not belong to the traveling wave class and are not generalized to the case of a nonzero velocity. But, to simplify the analysis, as was done in other studies [4-7], we consider the stationary case (zero-speed soliton).

Solutions are found in the form of radially symmetric solitons, which are functions of the form $\varphi(r)=$ $=A /\left(1+\alpha r^{2}\right)^{n}$, where $0<n \leq 1$ is any real number. The influence of a combination of the powers of nonlinearities and the spatial-dimension parameter on the solution obtained is analyzed. It is shown that a consistent increase in the powers of the nonlinearity in the equation leads to a decrease in the exponent $n$ regardless of the spatial dimension of the problem. As the dimensionality of space increases, the soliton is narrowed, and its amplitude increases.

\section{General Formulation and Solution of the Problem}

Soliton problems in nonlinear physical systems are usually reduced to a nonlinear Schrödinger equation with power nonlinearity. As a rule, they arise from equations of the following general form:

$\Delta \psi+\psi G(\psi)+\varepsilon \psi=0$.

In this case, the wave function is constructed in the form of a plane wave with modulated amplitude: $\psi(\mathbf{r})=\varphi(\mathbf{r}) e^{i \mathbf{k} \cdot \mathbf{r}}$. This leads to a redefinition of the eigenvalue $\varepsilon$ due to the dispersion of the energy term. The equation itself assumes the form:

$\Delta \varphi+\varphi g(\varphi)+\lambda \varphi=0$.

Further, the function $g(\varphi)$ is expanded in a series in powers of $\varphi$. Now, this can lead to another overdetermination of the parameter $\lambda$. To determine the modulating factor $\varphi(\mathbf{r})$, there are equations, which can be reduced in the radially symmetric representation to the form of the generalized nonlinear Schrödinger equation:

$\frac{\partial^{2} \varphi}{\partial r^{2}}+\frac{\beta}{r} \frac{\partial \varphi}{\partial r}+g_{1} \varphi^{a}-g_{2} \varphi^{b}+L \varphi=0$.
The generality of Eq. (2) relative to the ones usually considered is reduced to two circumstances. The first circumstance is the presence of a factor $\beta=$ $=0,1,2$, respectively, for $1 \mathrm{D}-(\beta=0), 2 \mathrm{D}-(\beta=1)$, and 3D-spaces $(\beta=2)$. This possibility of the simultaneous consideration of all three spatial dimensions in a solution is a specific feature of the radially symmetric consideration. The second circumstance is the presence of two nonlinear power terms: $g_{1} \varphi^{a}$ and $g_{2} \varphi^{b}$ (but not one, which is cubic, as usual). The coefficients $g_{1}$ and $g_{2}$ together with the coefficient $L$ depend on the parameters of the particular problem under consideration. At the same time, $a$ and $b$ in these terms are not given and, in the process of constructing a solution, can be made consistent with it. The presence of two nonlinear terms in Eq. (2) allows us to find analytical solutions for a number of combinations of powers $a, b$, which refer to real physical situations and will be considered further. The combination of signs at the coefficients $g_{1}, g_{2}$ ensures the maximum physical correctness of Eq. (2) and most of the specific situations considered below. There are, however, individual cases requiring either a combination of signs or even the absence of one of the nonlinear terms. They will be analyzed as well.

The solution of Eq. (2) is constructed in the form of a substitution:

$\varphi(r)=\frac{A}{\left(1+\alpha r^{2}\right)^{n}}$.

The parameters $A, \alpha$, and $n$ are determined from the condition that the left-hand side of Eq. (2) vanishes identically after the substitution of solution (3) into it. Such a substitution, after some transformations, leads to the relation

$$
\begin{aligned}
& -\frac{4 \alpha n(n+1)}{\left(1+\alpha r^{2}\right)^{2}}+\frac{2 \alpha n(2 n+1-\beta)}{\left(1+\alpha r^{2}\right)}+ \\
& +\frac{g_{1} A^{a-1}}{\left(1+\alpha r^{2}\right)^{(a-1) n}}-\frac{g_{2} A^{b-1}}{\left(1+\alpha r^{2}\right)^{(b-1) n}}+L=0 .
\end{aligned}
$$

As can be seen, to convert the left-hand side of this equation to zero, it is necessary to require that the algebraic sum of the first and third terms, as well as the second and fourth terms, is zero. The parameter $L$ must also be assumed to be zero. As was shown in $[9-16]$, the condition $L=0$ for the quantum and solid-state problems leads to the determination of the eigenvalue of the problem under consideration, which corresponds to Eq. (2). This is due to the fact that, 


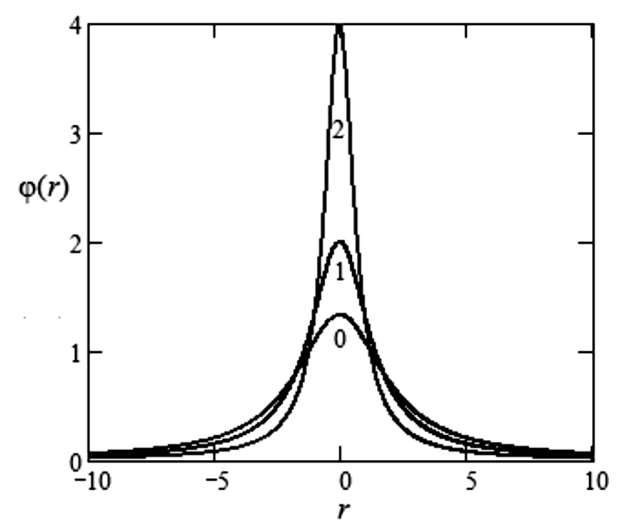

Fig. 1. Comparative graphs of solution (10) for $j=1$ and three values of the spatial-dimension parameter $\beta=0,1,2$. The plots on the graph correspond to the values $\beta$. The graphs show a special case $g_{1}=g_{2}=1$

in the simplest case, $L$ has a structure of the difference between this eigenvalue and the dispersion energy term, which determines the dynamic properties of a quasiparticle. In other problems, this term in Eq. (2) can be absent. Having in mind only such problems, we assume $L=0$ and require that the algebraic sum of the first and third, as well as the second and fourth terms, be zero.

As a result, the requirement of equality of the denominators yields two conditions that relate the powers $(a$ and $b)$ of the nonlinear terms and the power $n$ of the solution:

$a=\frac{2}{n}+1 ; \quad b=\frac{1}{n}+1$.

For the numerators, two conditions remain:

$-4 \alpha n(n+1)+g_{1} A^{a-1}=0 ;$

$2 \alpha n(2 n+1-\beta)-g_{2} A^{b-1}=0$.

With the help of these four conditions, it is necessary to determine three characteristics of solution (3): the amplitude $A$, growth rate $\alpha$, and power $n$. In this sense, system (4), (5) is overdetermined. However, as was mentioned above, one of the factors, the generalization of Eq. (2), is associated with the unconditionedness of the powers $a$ and $b$, and system (4), (5) becomes already underdetermined: five unknowns with four equations. In most physical problems, the powers $a, b$ are integer. This is due to the fact that the nonlinear terms in (2) are usually a consequence of the expansion in series of some potential energy that depends on the desired function $\varphi(r)$. From relations (4), it is clear that the integerity of the powers $a, b$ can be ensured, by assuming $n=1 / j, j=1,2,3, \ldots$. Then: $a=2 j+1, b=j+1$. In this case, Eq. (2) for each $j$ becomes

$\frac{\partial^{2} \varphi}{\partial r^{2}}+\frac{\beta}{r} \frac{\partial \varphi}{\partial r}+g_{1} \varphi^{2 j+1}-g_{2} \varphi^{j+1}=0$,

and solution (3) for this value $j$ reads

$\varphi(r)=\frac{A}{\left(1+\alpha r^{2}\right)^{1 / j}}$.

The coefficients $A$ and $\alpha$ are determined by Eqs. (5). Substituting $n=1 / j$ into them and solving them with respect to $A$ and $\alpha$, we obtain

$A=\left(\frac{2(1+j) g_{2}}{(2+(1-\beta) j) g_{1}}\right)^{1 / j} ; \alpha=\frac{j^{2}(1+j) g_{2}^{2}}{(2+(1-\beta) j)^{2} g_{1}}$.

Equation (6) and its solution in the form (7), (8) for different values $j=1,2,3, \ldots$, correspond to different physical situations. Some of them, the most common, will be discussed in the following sections.

\section{Generalized Cubic Nonlinearity $(j=1)$}

Assuming $j=1$ in (6)-(8), we get the equation

$\frac{\partial^{2} \varphi}{\partial r^{2}}+\frac{\beta}{r} \frac{\partial \varphi}{\partial r}+g_{1} \varphi^{3}-g_{2} \varphi^{2}=0$

Its solution and coefficients are as follows:

$\varphi(r)=\frac{A}{1+\alpha r^{2}}$,
$A=\frac{4 g_{2}}{(3-\beta) g_{1}} ; \quad \alpha=\frac{2 g_{2}^{2}}{(3-\beta)^{2} g_{1}}$.

Equation (9) is atypical of the solid-state problems related to a response of the crystal lattice to the excitation. This is due to the fact that, in them, the function $g(\varphi)$ of Eq. (1) usually depends not only on $\varphi$, but on $\varphi^{2}$ as well. When this function is expanded in a series, the structure $\varphi g(\varphi)$ cannot contain, in principle, a term $\varphi^{2}$. In other quantum problems, the problems of electrostatics, or problems of the interaction of a plasma with electromagnetic radiation, equations similar to (9) can be realized.

Figure 1 shows the comparative graphs of solution (10), (11) for all three spatial dimensions: $\beta=$ $0,1,2$.

ISSN 2071-0194. Ukr. J. Phys. 2018. Vol. 63, No. 10 
From the graphs in Fig. 1, it can be seen that, as the space dimension increases, the soliton is narrowed, and its amplitude increases.

An important property follows from solution (10) in the case under consideration. At infinity, the soliton has the asymptotics $\varphi(r) \sim 1 / r^{2}$. It is more typical of physical fields than the exponential one. In particular, it is typical of force characteristics such as the field strength. At the same time, the soliton is finite in the entire space, in particular at the point $r=0$.

\section{Generalized Nonlinearity of the Fifth Power $(j=2)$}

Assuming now $j=2$ in (6)-(8), we obtain the equation

$\frac{\partial^{2} \varphi}{\partial r^{2}}+\frac{\beta}{r} \frac{\partial \varphi}{\partial r}+g_{1} \varphi^{5}-g_{2} \varphi^{3}=0$

its solution

$\varphi(r)=\frac{A}{\left(1+\alpha r^{2}\right)^{1 / 2}}$,

and the coefficients

$A=\left(\frac{3 g_{2}}{(2-\beta) g_{1}}\right)^{1 / 2} ; \quad \alpha=\frac{3 g_{2}^{2}}{(2-\beta)^{2} g_{1}}$.

In solid-state problems, this equation can have a physical meaning for a certain symmetry of the function $g(\varphi)$ of Eq. (1). In these problems, as was already mentioned, the function $g(\varphi)$ depends not only on $\varphi$, but on $\varphi^{2}$. Thus, $g(\varphi) \equiv q\left(\varphi^{2}\right)$. In this case, if the expansion function $q(x)$ begins with a quadratic term [e.g., if $q(x)=1-\cos (x)$ ], then the expansion of the function $q\left(\varphi^{2}\right)$ would start with the term $\varphi^{4}$ [in the example, $\left.q\left(\varphi^{2}\right)=1-\cos \left(\varphi^{2}\right)\right]$. Solution (13) at infinity has the asymptotics $\varphi(r) \sim 1 / r$, which is also typical of physical fields. In particular, it corresponds to energy characteristics of a potential-type field.

\subsection{Cases of normal solution}

As is seen from the definition of the coefficients $A$ and $\alpha$, the trend for $1 \mathrm{D}$-spaces $(\beta=0)$ and $2 \mathrm{D}$-spaces $(\beta=1)$ is the same as in Fig. 1 . In particular, Fig. 2 shows the $3 \mathrm{D}$ picture of a distribution of the envelope of a soliton (the square of function (13)) in $2 \mathrm{D}$ spaces $(\beta=1)$.

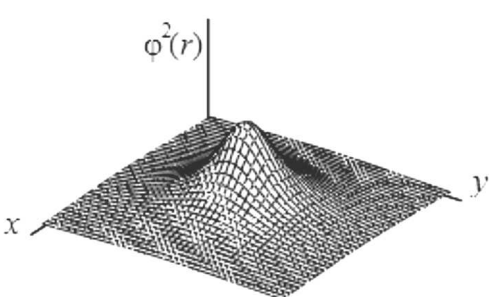

Fig. 2. The spatial form of a $2 \mathrm{D}$ soliton $(\beta=1)$ in a special case $g_{1}=g_{2}=1$. The amplitude $A$ and growth rate $\alpha$ have the numerical values $A=\sqrt{3}, \alpha=3$

For the generalized cubic nonlinearity $(j=1)$ considered in Sec. 3 , the shape of a $2 \mathrm{D}$-soliton $(\beta=1)$ will be qualitatively the same, as well as in cases that will be considered further.

\subsection{The case of specific solution}

The case of $3 \mathrm{D}$-soliton $(\beta=2)$ requires a separate consideration. As follows from definitions (14), this case is degenerate, since, at $\beta=2$, the coefficients $A$ and $\alpha$ formally become infinite. In order for them to be finite in the case where $\beta=2$ and $j=2$, $g_{2}=0$ should be assumed. This case (generalized fifth-power nonlinearity for $3 \mathrm{D}$ spaces $(\beta=2))$ was discussed in [17] in detail. It was shown there that one "free" parameter remains in the solution. In relations (14), this can be formally achieved, if we assume that if the parameter $g_{2}$ and differences $2-\beta$ tend to zero (above), their ratio $g_{2} /(2-\beta)$ tends to a certain number $\chi$. Then relations (14) formally take the form: $A=\sqrt{3 \chi / g_{1}}, \alpha=3 \chi^{2} / g_{1}$. Excluding the parameter $\chi$, it is possible to bring these two relations to a form, in which the growth parameter $\alpha: A=\sqrt[4]{3 \alpha / g_{1}}$.

\section{Generalized Nonlinearity of the Seventh Power $(j=3)$}

Here, Eq. (6) takes the form

$\frac{\partial^{2} \varphi}{\partial r^{2}}+\frac{\beta}{r} \frac{\partial \varphi}{\partial r}+g_{1} \varphi^{7}-g_{2} \varphi^{4}=0$

its solution (7) is determined by the relation

$\varphi(r)=\frac{A}{\left(1+\alpha r^{2}\right)^{1 / 3}}$,

and the coefficients $A$ and $\alpha$ defined in (8) are reduced to

$A=\left(\frac{8 g_{2}}{(5-3 \beta) g_{1}}\right)^{1 / 3}, \alpha=\frac{36 g_{2}^{2}}{(5-3 \beta)^{2} g_{1}}$. 
This case can be implemented, when the function $g(\varphi)$ in Eq. (1) has an even symmetry, and its expansion begins with a cubic term. For example, we can take $g(\varphi)=\cos \left(\varphi^{3 / 2}-1\right.$. In particular, the presence of the argument $\varphi^{3 / 2}$ says that this case is not implemented in solid-state problems, where, as was already noted, the function $g(\varphi)$ depends on $\varphi^{2}$. The asymptotics of the solution at infinity shows the unusual power of decrease: $\varphi(r) \sim 1 / r^{2 / 3}$.

\subsection{Cases of normal solution}

From the definitions of the coefficients $A$ and $\alpha$, it follows that, as in Section 4, we observe the same trend as in Fig. 1 for the $1 \mathrm{D}$-spaces $(\beta=0)$ and $2 \mathrm{D}$ spaces $(\beta=1)$, as the space dimensionality increases.

\subsection{Case of specific solution}

But there is one feature. For a 3D-space $(\beta=2)$, the soliton has a negative amplitude. This situation can be considered as a separate case. The negativity of the amplitude is not a separate case, if the square of the amplitude has a physical meaning. In addition, the amplitude of a 3D soliton for this case $(j=3, \beta=2)$ becomes positive, if $g_{2}<0$, that is, if $g_{2}=-\left|g_{2}\right|$. In this case, Eq. (15) takes the form $\frac{\partial^{2} \varphi}{\partial r^{2}}+\frac{2}{r} \frac{\partial \varphi}{\partial r}+g_{1} \varphi^{7}+\left|g_{2}\right| \varphi^{4}=0$. It can be implemented, when the function $g(\varphi)$ is not limited in the entire space of its argument, as, for example, if $g(\varphi)=\operatorname{ch}\left(\varphi^{3 / 2}\right)-1$.

\section{Generalized Nonlinearity of the Ninth Power $(j=4)$}

Now, Eq. (6) takes the form

$\frac{\partial^{2} \varphi}{\partial r^{2}}+\frac{\beta}{r} \frac{\partial \varphi}{\partial r}++g_{1} \varphi^{9}-g_{2} \varphi^{5}=0$.

Solution (7) is defined by

$\varphi(r)=\frac{A}{\left(1+\alpha r^{2}\right)^{1 / 4}}$.

The coefficients $A$ and $\alpha$ defined in (8) have now such a specific form

$A=\left(\frac{5 g_{2}}{(3-2 \beta) g_{1}}\right)^{1 / 4} ; \quad \alpha=\frac{20 g_{2}^{2}}{(3-2 \beta)^{2} g_{1}}$.

This case can be implemented with the same symmetry of the function $g(\varphi) \mathrm{n} \mathrm{Eq.} \mathrm{(1),} \mathrm{as} \mathrm{in} \mathrm{subsection}$
4.2. The only difference is that it could, for example, be the function: $g(\varphi)=\cos \left(\varphi^{2}\right)-1$. In this sense, the case under consideration can be of interest for solid-state problems. The asymptotics of the solution at infinity, as well as in the previous section, is atypical of physical fields, but it also causes interest in an unusual power of decrease: $\varphi(r) \sim 1 / r^{1 / 4}$. At the same time, the asymptotics $\varphi^{2}(r) \sim 1 / r^{1 / 2}$ can be already of interest in some cases and for physical fields.

\subsection{Cases of normal solution}

From the definition of the coefficient $A$, we can immediately conclude that the solitons in the cases of $1 \mathrm{D}(\beta=0)$ and $2 \mathrm{D}(\beta=1)$ spaces have the same properties as in all the previous sections (Fig. 1).

\subsection{Case of specific solution}

For a $3 \mathrm{D}$-space $(\beta=2)$, the amplitude becomes complex:

$A=\left(-\frac{5 g_{2}}{g_{1}}\right)^{1 / 4} \equiv \sqrt{i}\left(\frac{5 g_{2}}{g_{1}}\right)^{1 / 4}$,

where $i$ is the imaginary unit. Since the amplitude $\varphi(r)$ must be real here, it can be provided only by replacing the sign in front of the parameter $g_{2}$ and assuming, for example, $g_{2}=-\left|g_{2}\right|$ (as well as in the Section 5).

The remaining generalized nonlinearities, for which $j \geq 5$, are of little interest from the physical point of view and will not be considered here.

\section{Conclusions}

The generalized nonlinear Schrödinger equation has been analyzed in order to find radially symmetric soliton solutions. The equation contains a parameter that allows the analysis simultaneously for all three spatial dimensions, 1D, 2D, and 3D. In addition, it contains two nonlinear terms in different powers. We consider four combinations of powers, called generalized nonlinearities of the third, fifth, seventh, and ninth powers, which can be related to physically relevant problems. For the equation considered here, we find radially symmetric solutions that have powerlaw asymptotics at infinity $\sim 1 / r^{v}$, but are not exponentially decreasing. With an increase in the powers of nonlinearity of the equation in question, the power $v$ decreases. In all the cases considered, the solutions found for 1D-spaces (polymers) and 2D-spaces

ISSN 2071-0194. Ukr. J. Phys. 2018. Vol. 63, No. 10 
(graphenes, etc.) are normal from the physical point of view (they have a real and positive definite amplitude). For 3D-spaces, from the four options of nonlinearity considered, three options require a separate consideration. All of them are related to the need either to change the sign of the coefficient of a term with a lower power or even to turn this coefficient to zero. It is shown that, in all the cases considered, the soliton becomes narrower with an increase in the space dimensionality, and its amplitude grows.

1. S. Dutta, E.J. Mueller. Collective modes of a soliton train in a Fermi superfluid. Phys. Rev. Let. 118, 260402 (2017).

2. N. Taghizadeh, Q. Zhou, M. Ekici, M. Mirzazadeh. Soliton solutions for Davydov solitons in $\alpha$-helix proteins. Superlattices and Microstructures 102, 323 (2017).

3. L. Brizhik. Influence of electromagnetic field on solitonmediated charge transport in biological systems. Electromagn. Biol. Medicine 34, 123 (2015)

4. L.S. Brizhik, A.A. Eremko, B. Piette, W.J. Zakrzewski. Static solutions of a $D$-dimensional modified nonlinear Schrödinger equation. Nonlinearity 16, 1481 (2003).

5. L.S. Brizhik, A.A. Eremko, B. Piette, W.J. Zakrzewski. Spontaneous localization of electrons in lattices with nonlocal interactions. Phys. Rev. B 68, 104301 (2003).

6. L.S. Brizhik, A.A. Eremko, B. Piette, W.J. Zakrzewski. Spontaneous localization of electrons in two-dimensional lattices within the adiabatic approximation. J. Math. Phys. 44, 3689 (2003)

7. E.A. Kuznetsov, V.E. Zakharov. Nonlinear coherent phenomena in continuous media. In: Nonlinear Science at the Dawn of the 21st Century. Edited by P.L. Christiansen, M.P. Sorensen, A.C. Scott (Springer, 2000), Chap. 1 [ISBN: 3-540-66918-3].

8. Yu. Rapoport, V. Grimalsky, A. V. Lavrinenko, A. Boardman. Double resonant excitation of the second harmonic of terahertz raditation in dielectric-graphene layered metamaterials. J. Optics 19, 095104 (2017)

9. A.D. Suprun, L.V. Shmeleva. The centrally-symmetric solutions of electronic excitations of semiconductors in the conditions of relativistic like degeneracy of dynamical properties. Funct. Mater. 21, 69 (2014).
10. T.A. Davydova, A.I. Fishchuk. Upper hybrid nonlinear wave structures. Ukr. Fiz. Zh. 40, 487 (1995).

11. S. Hussain, S. Mahmood. Magnetosonic hump and dip solitons in a quantum plasma with Bohm potential effect. Phys. Plasmas 24, 032122 (2017).

12. R.Y. Chiao, E. Garmire, C.H. Townes. Self-trapping of optical beams. Phys. Rev. Let. 13, 479 (1964).

13. A.C. Scott. Dynamics of Davydov solitons. Phys. Rev. A 26, 578 (1982).

14. C.B. Muratov, V.V. Slastikov. Domain structure of ultrathin ferromagnetic elements in the presence of Dzyaloshinskii-Moriya interaction. Proc. Math. Phys. Eng. Sci. 473, 20160666 (2017).

15. L.S. Brizhik. Electron correlations in molecular chains. In: Correlations in Condensed Matter under Extreme Conditions. Edited by G.G.N. Angilella, A. La Magna (Springer, 2017), Chap. 15, p. 215.

16. A.D. Suprun. Self-accelerating Painleve-II soliton: A curious mathematical trick or fundamental physics? Funct. Mater. 9, 389 (2002).

17. A.D. Suprun. Two types of soliton solution of Schrödinger equation with the total nonlinearity of 5th degree. Funct. Mater. 8, 436 (2001).

Received 16.01.18

\section{А.Д. Супрун, Л.В.Шмельова}

ЦЕНТРАЛЬНО-СИМЕТРИЧНІ

СОЛІТОНИ ЗІ СТУПЕНЕВОЮ АСИМПТОТИКОЮ

ДЛЯ СЕРЕДОВИЩ РІЗНОЇ РОЗМІРНОСТІ

Р ез ю м е

У роботі проаналізовано аналітичні розв'язки радіальносиметричних нелінійних рівнянь Шредінгера з двома нелінійними доданками у різних ступенях для $1 \mathrm{D}, 2 \mathrm{D}$ і $3 \mathrm{D}$ просторів. Вони типові для рівнянь, у яких є два нелінійних доданки, а не один, як правило, кубічний. Важливою особливістю отриманих розв'язків є те, що вони виражаються не через гіперболічні функції, а через раціональні функції, скінченні у всьому просторі і мають ступеневу асимптотику на нескінченності. Отримані розв'язки істотно розширюють коло застосувань нелінійного рівняння Шредінгера. Окремі актуальні випадки загального розв'язку розглянуті в додатках. 